Annuaire suisse de politique de développement

26-2 | 2007

Financer le développement par la mobilisation des ressources locales

\title{
Microfinance : effets mitigés sur la lutte contre la pauvreté
}

Isabelle Guérin, Cyril Fouillet, Isabelle Hillenkamp, Olivier Martinez, Solène Morvant-Roux et Marc Roesch

\section{OpenEdition}

\section{Journals}

Édition électronique

URL : http://journals.openedition.org/aspd/127

DOI : $10.4000 /$ aspd. 127

ISSN : 1663-9669

Éditeur

Institut de hautes études internationales et du développement

Édition imprimée

Date de publication : 1 novembre 2007

Pagination : 103-119

ISBN : 978-2-88247-068-3

ISSN : 1660-5934

Référence électronique

Isabelle Guérin, Cyril Fouillet, Isabelle Hillenkamp, Olivier Martinez, Solène Morvant-Roux et Marc

Roesch, «Microfinance : effets mitigés sur la lutte contre la pauvreté », Annuaire suisse de politique de développement [En ligne], 26-2 | 2007, mis en ligne le 19 juin 2009, consulté le 08 septembre 2020. URL : http://journals.openedition.org/aspd/127 ; DOI : https://doi.org/10.4000/aspd.127 


\title{
Microfinance:
}

\section{effets mitigés sur la lutte contre la pauvreté}

\author{
Isabelle Guérin, Cyril Fouillet, Isabelle Hillenkamp, \\ Olivier Martinez, Solène Morvant-Roux et Marc Roesch*
}

L

a nobélisation de Muhammad Yunus et de la Grameen Bank suscite un consensus médiatique sans précédent sur les vertus supposées de la microfinance en matière de lutte contre la pauvreté et de réduction des inégalités. «Un instrument fort efficace de libération des pauvres, en particulier des femmes » : c'est ainsi que les membres du jury du Nobel justifient leur choix en considérant qu'il ne saurait y avoir de paix sans réduction de la pauvreté, et il a été démontré, disent-ils, que le microcrédit y participait activement.

Dans quelle mesure et à quelles conditions la microfinance contribue effectivement à la lutte contre la pauvreté ? Des recherche menées pendant plusieurs années dans des régions très diverses et un travail intensif de collecte des données montrent que la microfinance est utile, mais qu'elle permet difficilement de lutter contre la pauvreté et que, dans certains contextes, elle peut même contribuer à renforcer certaines formes d'inégalités.

La légitimité de la microfinance ne fait aucun doute: dans un contexte de financiarisation croissante, les pauvres plus que quiconque ont besoins de services financiers. En pratique toutefois, les effets sont beaucoup plus limités que ne le clament les discours actuels sur la microfinance ${ }^{1}$, pour deux raisons principales. La première est une vision naïve, simpliste et trop économique des problèmes de pauvreté et d'inégalités. La seconde vient de l'offre de microfinance, caractérisée par des services encore peu adéquats, un nombre croissant d'acteurs non seulement inexpérimentés mais dont les motivations sont parfois douteuses et des inégalités territoriales extrêmement fortes. Par ailleurs, on observe bel et bien des effets positifs, mais ils sont souvent inattendus: les services de microfinance ne sont pas consommés passivement par leurs clients, mais traduits, interprétés et ensuite utilisés de manière souvent subtile et stratégique en empruntant des voies parfois contradictoires avec les objectifs des promoteurs de ces services.

* Isabelle Guérin, chercheuse à l'Institut de recherche pour le développement (IRD, Laboratoire population environnement développement [LPED]), France, et à l'Institut français de Pondichéry (IFP), Inde.

Cyril Fouillet, doctorant au Laboratoire d'économie de la firme et des institutions (Université Lyon 2), rattaché au LPED et à l'IFP, Inde.

Isabelle Hillenkamp, doctorante à l'Institut universitaire d'études du développement (IUED), Genève. Olivier Martinez, doctorant au LPED (Université de Provence Aix-Marseille 1), France.

Solène Morvant-Roux, chercheuse associée à l'IRD (LPED), France.

Marc Roesch, chercheur au Centre de coopération internationale en recherche agronomique pour le développement, en poste d'accueil à l'IRD (LPED) et à l'IFP, Inde.

1 Le dernier rapport de la Campagne du microcrédit affirme par exemple que le microcrédit «est l'un des outils les plus performants pour résoudre le problème de la pauvreté dans le monde» (S. DaleyHarris, Etat de la campagne du Sommet du microcredit. Rapport 2006, 2006, <http://www.microcreditsummit.org/french/pubs/reports/socr/2006/SOCR06_fr.pdf> [consultation: $1^{\text {er }}$ février 2007], p. 1). 
Institutions internationales (du type Banque mondiale, Programme des Nations unies pour le développement, Banque asiatique de développement, Organisation des Nations unies pour l'alimentation et l'agriculture, etc.), agences multilatérales ou bilatérales de coopération (du type Agence française de développement, KFW allemande, DANIDA danois, etc.), gouvernements nationaux mais aussi collectivités locales (régions, communes, etc.) et enfin organisations non gouvernementales (ONG du Nord ou internationales mais aussi ONG locales financées par des bailleurs de fonds): l'ensemble des acteurs impliqués dans le développement intègrent désormais la microfinance dans leurs agendas. Le «marché», via les institutions financières et bancaires, commence également à s'y intéresser sérieusement. Cette frénésie d'acteurs n'est hélas pas nécessairement compatible avec la mise en œuvre effective d'une mission de lutte contre la pauvreté.

Du côté des institutions «marchandes» (établissements bancaires, institutions financières, fonds de placement, etc.), l'accent mis sur les exigences de rentabilité financière est difficilement compatible avec un objectif de lutte contre la pauvreté ${ }^{2}$. Citons par exemple la concentration sur les franges de clientèle et les territoires les plus rentables, la focalisation sur les volumes octroyés aux dépens de la qualité des services, les taux d'intérêt parfois supérieurs à la rentabilité des activités financées et, dans certains cas, des méthodes d'octroi des prêts proches de la vente forcée et des méthodes de recouvrement des prêts dignes des usuriers les plus tenaces ${ }^{3}$. Du côté des organisations à but non lucratif, il existe aussi de nombreux risques: privilégier la microfinance aux dépens d'actions qui seraient pourtant prioritaires (défense des droits, santé, éducation). Ces dérives ont été constatées par exemple en Inde ${ }^{4}$, au Bangladesh ${ }^{5}$ et au Sri Lanka ${ }^{6}$. Nombreuses sont les organisations qui se sont engagées dans la microfinance sans avoir les moyens humains de le faire. Insistons sur la complexité et la diversité des compétences exigées pour une offre de microfinance de qualité. Le premier défi consiste à combiner de multiples savoir-faire, relevant tout autant de la finance et de la gestion que du travail social. Il est également essentiel d'avoir une connaissance très fine des populations et des territoires d'intervention, condition rarement compatible avec les délais imposés par les bailleurs de fonds

2 D'autre part, il est important de ne pas confondre pauvreté et exclusion financière. Dans de nombreux pays, la population n'ayant pas accès à des services financiers ne recouvre pas seulement la population dite pauvre. L'image d'une pyramide permet de comprendre la situation: à sa base, les populations en situation permanente de pauvreté et dont les capacités d'autoemploi sont souvent très faibles; au-dessus, des personnes en situation de plus ou moins grande précarité avec des revenus instables; enfin, en haut de cette pyramide, les populations financièrement intégrées constituées de petits commerçants, de salariés ou encore d'importants propriétaires terriens. Pour chacune de ces populations, les besoins en services financiers s'avèrent très différents et nécessitent une offre adaptée. Voir: J.-M. Servet, Banquiers aux pieds nus. La microfinance, Paris, Odile Jacob, 2006.

3 Voir: C. Fouillet, I. Guérin, S. Morvant-Roux, M. Roesch et J.-M. Servet, «Le microcrédit au péril du néolibéralisme et de marchands d'illusions. Manifeste pour une inclusion financière socialement responsable », Revue du MAUSS, à paraître.

4 Voir: I. Guérin, C. Fouillet et J. Palier, «La microfinance indienne peut-elle être solidaire?», Revue Tiers-Monde, à paraître.

5 Voir: J.L. Fernando (ed.), Microfinance: Perils and Prospects, London, Routledge, 2006. K. Stiles, «International Support for NGOs in Bangladesh: Some Unintended Consequences », World Development, vol. 30, n 5, 2002, pp. 835-846.

6 Voir: J.L. Fernando (ed.), op. cit. 
ou avec les exigences de rentabilité financière. Un autre défi consiste à répondre simultanément aux besoins des populations, d'une complexité et d'une diversité considérables, et aux contraintes organisationnelles et institutionnelles du contexte d'intervention. La législation bancaire, par exemple, est souvent inadéquate et oblige les organisations de microfinance à délaisser certains services pourtant essentiels (par exemple la mobilisation d'épargne). Ce jonglage permanent entre des exigences parfois contradictoires suppose une capacité considérable d'adaptation et d'innovation, qualités qui font défaut à nombre d'organisations, faute de souplesse interne mais aussi du fait de bailleurs et de financeurs pressés et focalisés sur des résultats quantitatifs.

\section{Des services encore mal adaptés}

En dépit d'injonctions récurrentes en faveur de l'innovation et de la diversification des services de microfinance ${ }^{7}$, force est de constater aujourd'hui l'incapacité de nombreuses organisations de microfinance à répondre de manière efficace à une demande qui paraît multiple et complexe. Si les impacts sont mitigés, c'est souvent parce que les services répondent encore mal à la demande. Du côté du microcrédit, les services sont souvent trop rigides, tant en termes de montants et de modalités de remboursement que de durée; les délais d'octroi sont souvent trop longs pour répondre efficacement à l'urgence des besoins. Du côté de l'épargne, on sous-estime souvent la complexité des attentes et des besoins des populations pauvres. Contrairement aux populations des pays $\mathrm{du}$ Nord, les personnes ne sont pas seulement à la recherche de rémunération ou de sécurité ${ }^{8}$. Les motifs de liquidité (pouvoir disposer de son épargne facilement et rapidement), de proximité (pouvoir épargner immédiatement en cas de surplus de liquidité) et d'anonymat (en particulier pour les femmes), la recherche de mécanismes d'incitation et d'épargne «forcée» (qui se comprennent aisément dans un contexte où les sollicitations de l'entourage sont permanentes, mais aussi où l'absence de prélèvements obligatoires [cotisations sociales] rend tout effort d'épargne volontaire inefficace et insuffisant) sont autant de motivations essentielles, mais auxquelles les services actuels répondent encore très mal ${ }^{9}$.

7 Au milieu des années 1990, David Hulme et Paul Mosley plaidaient déjà en faveur d'une diversification des services financiers, arguant de la nécessité d'adapter les produits à des besoins multiples et de faire preuve de souplesse, en particulier pour les plus pauvres (D. Hulme and P. Mosley, Finance against Poverty, vol. 1, London, Routledge, 1996). Le thème de l'innovation a été largement repris par la Campagne du microcrédit en 2002 (A. Simanovitz and A. Walter, «Ensuring Impact: Reaching the Poorest while Building Financially Self-sufficient Institutions, and Showing Improvement in the Lives of the Poorest Women and Their Families », in S. Daley-Harris (ed.), Pathways out of Poverty: Innovations in Microfinance for the Poorest Families, Bloomfield, CT, Kumarian Press, 2002, pp. 1-90). Sur la nécessité d'innover, voir également J. Morduch, "The Microfinance Promise», Journal of Economic Literature, vol. 37, n 4, 1999, pp. 1569-1614, ainsi qu'un numéro spécial du Journal of International Development $\left(\mathrm{n}^{\circ} 14,2002\right)$.

8 Voir: J.-M. Servet Epargne et liens sociaux. Etudes comparées d'informalités financières, Paris, Association d'économie financière, 1995

9 Pour des analyses empiriques concrètes, voir: I. Guérin, «Women and Money: Multiple, Complex and Evolving Practices», Development and Change, vol. 37, $\mathrm{n}^{\circ}$ 3, 2006, pp. 549-570. M. Schreiner and R. Vonderlack, «Women, Microfinance and Savings: Lessons and Proposals», Development in Practice, vol. 12, nº 5, 2002, pp. 602-612. 
Les inégalités territoriales de l'offre de microfinance sont considérables. Audelà des inégalités entre continents (concentration extrême en Asie, développement relatif en Amérique latine et «retard» avéré en Afrique) ${ }^{10}$, les espaces nationaux sont eux aussi marqués par de très fortes disparités. L'offre a souvent tendance à se concentrer dans les territoires les plus riches, avec deux conséquences: un renforcement des inégalités territoriales préexistantes, mais aussi des risques de saturation de l'offre et de surendettement des clients. Dans nombre de pays africains (c'est particulièrement évident au Bénin - voir cidessous), les zones rurales sont souvent désertées alors qu'elles ont bien plus de pauvres, tandis que certains espaces urbains font l'objet d'une concurrence intense largement propice au surendettement ${ }^{11}$. Des initiatives de coordination de nature diverse (formelle ou informelle, souvent lancées par des bailleurs de fonds) existent ici et là: c'est le cas par exemple au Pakistan, au Bangladesh, au Mali, au Ghana, en Ouganda, en Tanzanie et en Bosnie ${ }^{12}$. Certains pays mettent en place une politique volontariste visant à corriger l'inégalité de l'offre. Citons le cas du Maroc, dans le cadre de l'Initiative nationale pour le développement humain, et celui de l'Inde, avec le programme «Inclusion» de la Reserve Bank for India ${ }^{13}$. Citons également le cas du Mexique, où la marginalisation des zones rurales des Etats les plus pauvres de la République en matière d'offre de microfinance était telle que l'Etat fédéral, appuyé par la Banque mondiale, y a mis en place à partir de 2001 un programme d'appui et de structuration de services d'intermédiation financière ${ }^{14}$. En dépit de ces quelques exemples, qui sont d'ailleurs souvent fragiles et éphémères, les tentatives de coordination et de régulation de l'offre relèvent encore davantage de l'exception que de la règle.

\section{Les inégalités nationales et régionales: I'exemple indien}

Avec un premier cycle de croissance qui a vu passer le nombre de clients de quelques centaines à plusieurs millions, le sous-continent indien apparaît aujourd'hui comme l'une des régions les plus dynamiques du développement de la microfinance. Cependant, malgré des résultats remarquables en termes de

10 Voir: C. Lapenu and M. Zeller, Distribution, Growth, and Performance of Microfinance Institutions in Africa, Asia, and Latin America, FCND Discussion Paper, $\mathrm{n}^{\circ} 114$, Washington, International Food Policy Research Institute, June 2001.

11 Voir: C. Fouillet, La microfinance serait-elle devenue folle? Crise en Andhra Pradesh (Inde), Bulletins d'information du mardi (BIM), Pôle microfinancement (CIRAD/GRET), 25 avril 2006. M. Roesch, Des dettes jusqu'à ne plus en vivre, Bulletins d'information du mardi (BIM), Pôle microfinancement (CIRAD/GRET), 11 avril 2006. U. Vogelgesang, «Microfinance in Times of Crisis: The Effects of Competition, Rising Indebtedness, and Economic Crisis on Repayment Behavior», World Development, vol. $31, \mathrm{n}^{\circ} 12$, pp. 2085-2114.

12 Voir: Groupe consultatif d'assistance aux pauvres (CGAP), «La coordination entre bailleurs de fonds à l'intérieur d'un pays », in I. Guérin et J.-M. Servet (dir.), Exclusion et liens financiers. Rapport du Centre Walras 2003, Paris, Economica, 2003, pp. 342-361

13 Voir: Rakesh Mohan, Economic Growth, Financial Deepening and Financial Inclusion, Annual Bankers' Conference 2006, Hyderabad, 3 November 2006, <http://www.rbi.org.in/scripts/BS_SpeechesView.aspx ?Id=310> (consultation: 5 juin 2007).

14 Voir: E. Arredondo Casillas, Estudio para el Desarrollo y Fortalecimiento de Instituciones Financieras Rurales en las Regiones Marginadas de Oaxaca, SAGARPA; Banco mundial, 2001. 
croissance, nos recherches montrent que ce mouvement s'est accompagné de fortes inégalités spatiales.

Carte 1: Distribution par départements des foyers participant à un self-help group (groupe d'emprunteurs) sur le territoire national indien, 2005-2006 (en pourcentage, méthode des seuils naturels) ${ }^{\mathrm{a}}$

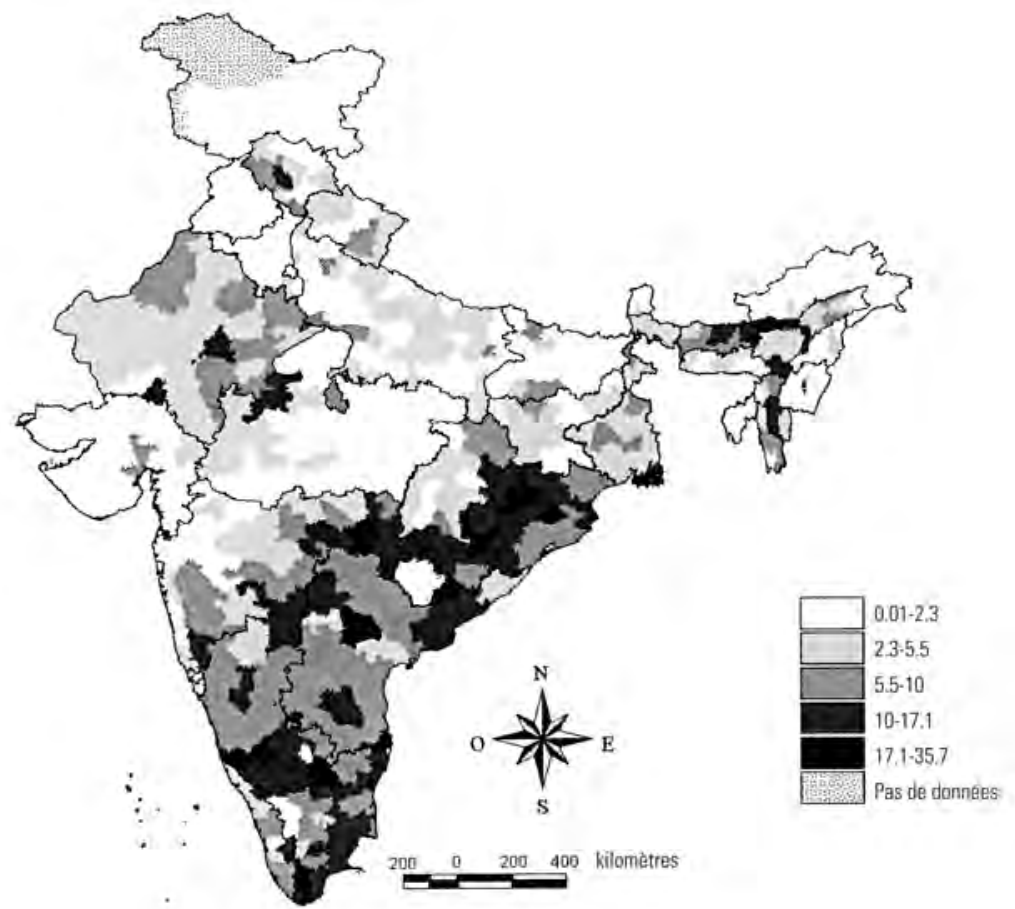

Source: C. Fouillet, Construction sociale, économique et spatiale de la microfinance: l'exemple de l'Inde, thèse de doctorat en sciences économiques et sociales, Université Lumière Lyon 2, à paraître.

a La méthode des seuils naturels (ou encore seuils observés) est une méthode de discrétisation manuelle qui se base sur l'observation d'un histogramme de fréquence (ou graphe de dispersion). Cette méthode, permettant de respecter la distribution des données, prend en compte les discontinuités de la série observée.

Au niveau national, les taux de croissance annuels moyens sont considérables (entre mars 1993 et mars 2006, le nombre des groupes d'emprunteurs a augmenté de $82 \%$ par an et les montants des prêts octroyés de $110 \%$ par an), mais les moyennes dissimulent une grande disparité selon les régions au profit des Etats du sud, qui figurent parmi les Etats les plus riches du territoire indien (Etats de l'Andhra Pradesh, du Karnataka, du Kerala, du Tamil Nadu et des Territoires de Pondichéry). Même si ces inégalités tendent à se réduire, en 20052006, les Etats du sud réunissaient à eux seuls $44 \%$ des groupes d'emprunteurs financés et près de $74 \%$ du montant total des prêts distribués sur le territoire indien (en 2000, ces proportions étaient de $67 \%$ et $78 \%$ ).

Cette forte hétérogénéité spatiale ne se limite pas seulement au niveau national mais se retrouve également au sein des Etats (voir cartes 1 et 2). Dans l'Etat du Tamil Nadu par exemple, alors que les districts de Dharmapuri, Tirunelveli et Thiruvallur affichent, pour l'année financière 2005-2006, un pourcentage de 
microfinanciarisation ${ }^{15}$ de $21,6 \%, 16,9 \%$ et $16 \%$, ce même taux reste très faible dans les districts de Nilgiris, Coimbatore et Karur $(0,8 \%, 1,6 \%$ et 3,9\% respectivement).

Par ailleurs, la comparaison en termes de distribution spatiale de l'offre de microfinance et de l'offre bancaire montre que l'un des effets de la microfinance a été de compenser le mouvement de débancarisation engendré par le processus de libéralisation du secteur financier et non, comme annoncé par les autorités indiennes, de combler le fossé entre l'offre et la demande de services financiers de la population rurale. En d'autres termes, on peut dire que jusqu'à présent, la microfinance a joué un rôle de filet de protection face aux politiques de libéralisation et aux mesures économiques austères qui les accompagnent.

\section{Carte 2: Distribution par départements des foyers participant à un self-help group (groupe d'emprunteurs) dans l'Etat du Tamil Nadu, 2005-2006) (en pourcentage, méthode des seuils naturels)}
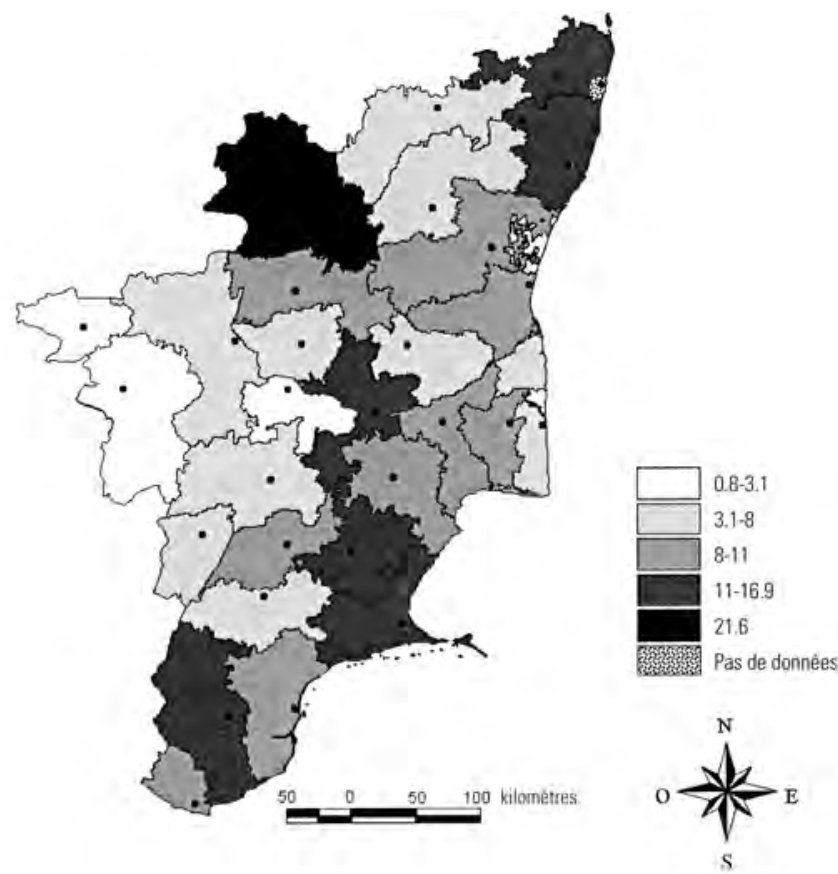

Source: C. Fouillet, Construction sociale, économique et spatiale de la microfinance: l'exemple de l'Inde, thèse de doctorat en sciences économiques et sociales, Université Lumière Lyon 2, à paraître.

\section{Les inégalités entre les zones rurales et urbaines: l'exemple béninois}

Depuis plus d'une quinzaine d'années, le Bénin a connu une forte croissance de l'offre de microfinance. Dès le début des années 1990, de grands programmes d'appui lancés par la Banque mondiale ont favorisé l'émergence d'organisations

15 Proportion de foyers dont une personne au moins est impliquée dans un groupe d'emprunteurs financé dans l'année. 
très diverses (en particulier en matière de taille). Comme pour l'Inde, la forte croissance de l'offre s'est faite de manière très inégale d'un point de vue géographique, avec d'une part une forte concentration dans les zones urbaines, moins pauvres que les campagnes, et d'autre part une forte concentration dans des zones où la finance informelle est déjà très dynamique, entraînant donc ici des risques de surendettement des clients mais aussi de déstructuration de pratiques financières informelles (en particulier les tontines) ${ }^{16}$.

Les inégalités spatiales de la microfinance interviennent à différentes échelles. Sur le plan national, elles révèlent une disproportion dans la répartition des points de service au niveau des 12 départements béninois (voir carte 3). Le département du Littoral, qui correspond à l'agglomération de Cotonou, en est l'exemple le plus frappant puisqu'il regroupe environ $20 \%$ de l'offre en services de microfinance, au détriment d'autres départements comme la Donga ou le Couffo, beaucoup plus pauvres et surtout nettement moins urbanisés.

\section{Carte 3: Répartition par départements des points de service des 26 principales IMF opérant sur le territoire national béninois (2003-2004)}
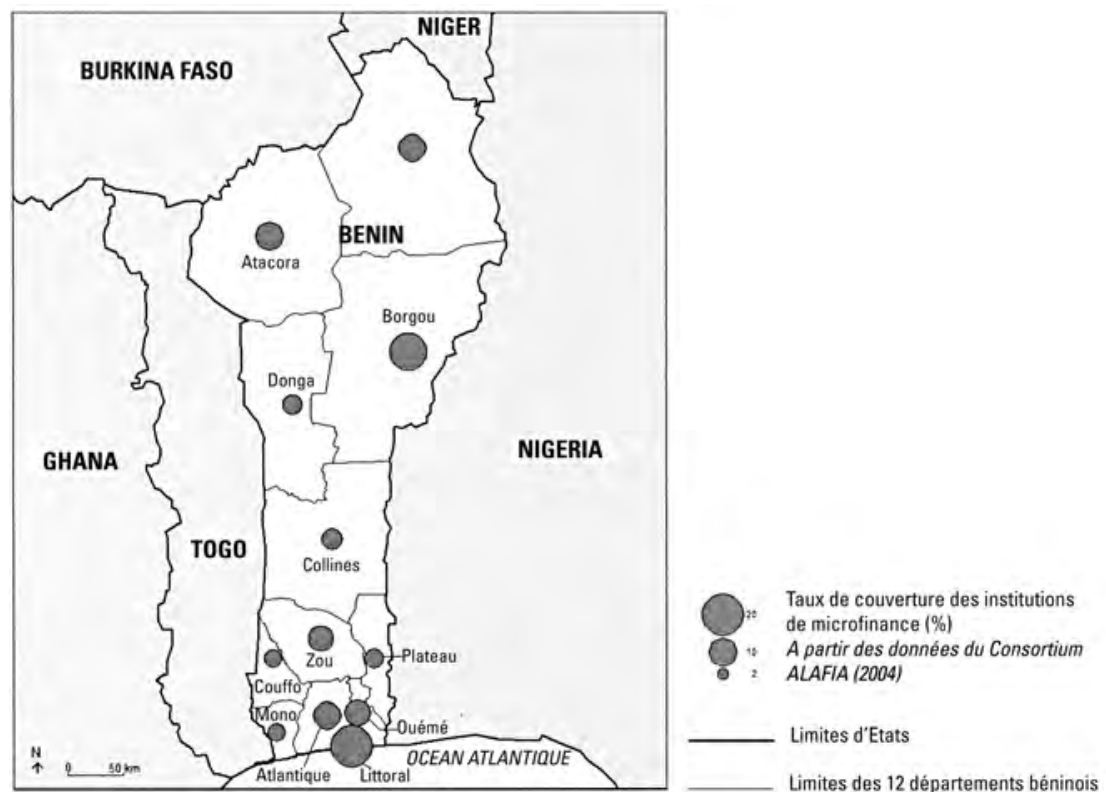

Source: carte réalisée par 0. Martinez (LPED/RD) à partir des données du Consortium ALAFIA, créé en 2000 et regroupant les 26 plus importantes IMF présentes au Bénin. En termes d'inégalités territoriales, l'image qui en ressort est donc partielle mais permet cependant de mettre en évidence certains phénomènes de concentration.

Un second niveau d'analyse, plus localisé, permet de mettre en évidence les disparités spatiales qui existent entre espaces urbains, périurbains et ruraux, profitant très largement aux premiers dans la mesure où ils rassemblent une clientèle

16 Voir: O. Martínez, Microfinance et territoires dans le Sud-Est béninois. Approche en termes de risque au travers d'une vision discriminante de l'espace, LPED, Marseille, Université de Provence AixMarseille 2, à paraître, 13 p. 
potentiellement plus nombreuse sur des surfaces plus restreintes. En considérant les seuls départements du Littoral (agglomération de Cotonou), de l'Atlantique (le plus peuplé des 12 départements), de l'Ouémé (abritant la capitale et seconde ville du pays, Porto-Novo) et du Borgou (dont le chef-lieu de département est Parakou, troisième ville du pays), on totalise $51 \%$ des points de service présentés sur la carte - pour environ $40 \%$ de la population nationale. L'attractivité des espaces urbains est donc en partie confirmée, notamment si on met en parallèle le taux de couverture de ces 4 départements avec ceux du Couffo ou des Collines, respectivement 4 et $5 \%$, nettement plus ruraux. Si la répartition des taux de couverture semble correspondre aux poids démographiques de chaque département, elle n'est en rien proportionnelle à ceux-ci et encore moins aux niveaux de pauvreté des départements.

Enfin, à une échelle intra-urbaine, ce sont souvent les zones économiquement dynamiques - comme les marchés et leurs périphéries - qui affichent les plus fortes densités de points de service et illustrent une nouvelle fois l'éloignement relatif de l'objectif de réduction de la pauvreté mis en avant par la plupart des institutions de microfinance (IMF) implantées au Bénin.

\section{Un impact limité et fragile sur la création d'emplois}

Nos analyses, basées sur plusieurs années d'observations, montrent que l'impact de la microfinance en termes de création d'emplois est en l'état actuel des choses négligeable. Contrairement aux présupposés largement véhiculés par un grand nombre d'acteurs, d'institutions et de réseaux (en particulier la Campagne du microcrédit), il est illusoire d'espérer que les pauvres puissent se transformer en microentrepreneurs, pour plusieurs raisons.

Une première raison concerne l'environnement économique dans lequel évoluent ces microentrepreneurs, caractérisé par des filières d'activités et des marchés locaux hyperhiérarchiques et monopolistiques. De ce fait, la faiblesse ou l'absence de rentabilité des activités financées par le microcrédit, la non-compétitivité face aux produits manufacturés, l'absence ou l'insuffisance de demande locale sont des facteurs d'échecs récurrents.

Ensuite, créer une entreprise nécessite des savoir-faire, un réseau relationnel et l'accès à l'information. Ces facteurs font bien souvent défaut à une population marginalisée économiquement et socialement. L'attachement psychologique et social au salariat ou à l'agriculture est négligé alors qu'il peut être très fort et entraîner des réticences bien compréhensibles face à une initiative nouvelle et une prise de risque individualisée.

Les montants limités des prêts octroyés ou encore le manque de flexibilité dans les méthodologies de remboursement, inadaptées aux fluctuations des revenus, sont une autre raison. Comme évoqué plus haut, en dépit d'un discours récurrent sur la nécessité d'introduire plus de flexibilité dans les services de microfinance, en pratique les services évoluent très peu dans ce sens. On comprend ainsi mieux pourquoi la majorité des microcrédits sont en fait utilisés pour des usages «non productifs » (c'est-à-dire pour des activités qui ne généreront pas directement de revenu). 
Insistons néanmoins sur la diversité des contextes locaux et des potentialités qui en résultent en matière de création d'emploi et de revenu via la microfinance. Lorsque certaines conditions sont réunies (offre de qualité, mais aussi forte densité de population ayant une capacité à consommer, spécialisation dans des produits dans des filières intégrées bénéficiant de la garantie d'achat, regroupement de producteurs facilitant l'accès au marché, etc.), alors l'impact peut être important, tant à l'échelle familiale que sur le plan du développement local ${ }^{17}$.

Les trois études de cas ci-dessous illustrent la diversité des impacts en fonction des contextes socioéconomiques locaux et de la nature de l'offre.

\section{L'impact limité sur la création d'emplois: un exemple indien}

Une étude longitudinale (2003-2006) menée par des chercheurs de l'Institut de recherche pour le développement et de l'Institut français de Pondichéry auprès de 400 clients d'une organisation de microfinance implantée au Tamil-Nadu (sud de l'Inde) met en évidence un impact marginal en termes de création d'emplois, et cela en dépit de mesures complémentaires (formation, création de coopérative $)^{18}$. La population cible se caractérise par une extrême vulnérabilité. Les revenus sont très faibles mais aussi très irréguliers, la plupart des personnes étant travailleurs agricoles journaliers pour le compte d'autrui, et l'endettement (avant microfinance) est très élevé, représentant en moyenne un an de salaire. Les familles jonglent en permanence avec de multiples formes de financement (jusqu'à six à huit créanciers), et une part importante de leurs revenus est consacrée au remboursement de prêts. Cette étude montre que, même après plusieurs années, $80 \%$ environ du montant des crédits de microfinance et bancaires sont utilisés pour des dépenses dites «non productives», c'est-à-dire ne générant aucun revenu direct: en priorité le remboursement d'anciennes dettes $(25 \%$ en moyenne) et les dépenses de santé (19\% en moyenne). Parmi les clients ayant trois ans d'ancienneté, $15 \%$ environ ont réussi à créer une activité génératrice de revenu grâce au microcrédit (les activités les plus fréquentes sont les petits commerces, la vente ambulante, la couture et la broderie). Les revenus qui en sont issus sont limités et irréguliers, et les activités restent fragiles; si la proportion de $15 \%$ reste stable, le profil des entreprises évolue: certaines se créent, d'autres ferment leurs portes. Les clients sont plus nombreux à investir dans l'élevage laitier sous l'impulsion des ONG ou des programmes gouvernementaux. L'impact, ici encore, est néanmoins limité en termes de revenu. Une analyse fine de la rentabilité de l'activité montre qu'elle est déficitaire pendant toute la période de remboursement du prêt, puis bénéficiaire six ou sept mois par an et déficitaire le reste du temps. Dans le meilleur des cas (bonne gestion et bonnes compétences techniques), le lait contribue pour $10 \%$ au revenu annuel d'un ménage d'ouvrier agricole. L'analyse d'un programme spécifique (Milch

17 Voir: F. Doligez, «Microfinance et dynamiques économiques: quels effets après dix ans d'innovations financières?», Revue Tiers Monde, vol. 43, n 172, 2002, pp. 783-809. B. Wampfler, Les conventions de microfinance: un outil de développement socialement durable. Une approche institutionnaliste, mémoire de travaux en vue de l'habilitation à diriger les recherches, Université de Saint-Quentin-enYvelines, 2004.

18 Voir: M. Roesch, I. Guérin, S. Kumar, M. Thanuja, Venkatasubramanian and Ponnarasu, Impact Assessment of the Peblisa Project in Tamil Nadu, Report for the International Labour Organisation, Pondicherry, French Institute of Pondicherry, 2006. 
Cow Credit) dans deux villages (l'un avec une population peu familière de la production de lait, l'autre en produisant déjà un peu) montre que les échecs sont liés au manque d'expérience en matière d'élevage bovin et à l'absence de formation et de suivi. Outre les difficultés financières que vivent alors les familles, l'échec a également tendance à décourager ces dernières de se lancer dans tout autre investissement qui leur serait proposé. Ainsi, dans le premier village (une majorité de bénéficiaires sans expérience), il reste $58 \%$ des animaux (les autres sont décédés ou ont été vendus), dont la moitié sont en production; dans le second (bénéficiaires plus expérimentés), $88 \%$ des animaux sont toujours présents. L'intérêt essentiel de cette production n'est pas la rentabilité économique, comme on pourrait s'y attendre, mais l'assurance de liquidités monétaires quotidiennes (vente de lait), le renforcement du patrimoine et donc de la sécurité familiale et, enfin, la contribution au prestige social familial.

\section{Le rôle prépondérant du contexte global dans l'évolution des revenus: un exemple mexicain}

Au Mexique, des recherches menées auprès de 240 emprunteurs d'une institution de microfinance (IMF) implantée dans des villages localisés dans des zones montagneuses et fortement dépendants de l'activité agricole, destinée soit à l'autoconsommation (maïs, haricot), soit à la vente (café), révèlent que la microfinance permet au mieux de stabiliser l'activité économique. Toutefois, des facteurs macroéconomiques comme la baisse des prix de vente du café (culture de rente dominante dans ces villages) depuis près de dix ans ont en fait un rôle déterminant qui limite fortement l'impact et les potentialités de la microfinance ${ }^{19}$. Une large partie des crédits (entre un tiers et la moitié) est investie dans l'activité agricole, notamment pour les dépenses liées à la main-d'œuvre lors des différentes étapes de la récolte. Mais les revenus tirés de cette activité n'augmentent pas pour autant. En l'absence d'opportunités locales de diversification des revenus, le microcrédit garantit la continuité de l'activité mais permet rarement d'en commencer une nouvelle ou d'accroître les revenus tirés des activités financées si le contexte local (demande solvable) ou global (prix de vente des produits agricoles) n'est pas propice. Dans le contexte étudié, la migration nationale temporaire et/ou le travail salarié restent les stratégies privilégiées, pour survivre au quotidien, mais aussi dans certains cas pour rembourser le microcrédit... Par ailleurs, dans les zones qui historiquement ne sont pas des zones à forte intensité migratoire, la migration vers les Etats-Unis est devenue la stratégie de diversification des sources de revenus la plus attractive. Malgré la présence prolongée du dispositif de microfinance (cinq ans), on observe depuis quelques années un net accroissement de la dynamique migratoire internationale. La microfinance ne constitue pas une alternative à la migration. Au contraire, en permettant aux migrants potentiels de financer une partie du voyage, elle intervient comme un facteur incitatif supplémentaire.

19 Voir: S. Morvant-Roux, Processus d'appropriation des dispositifs de microfinance: un exemple en milieu rural mexicain, thèse de doctorat en sciences économiques, Université Lumière Lyon 2, 2006. 
La Bolivie est sans aucun doute le pays d'Amérique latine où la microfinance est la mieux implantée. La clientèle se concentre parmi les propriétaires de microentreprises, et la microfinance représente aujourd'hui une part non négligeable de leurs sources de financement (près d'un tiers). On connaît pourtant mal les effets réels de la microfinance sur le tissu entrepreneurial bolivien. Les évaluations se sont longtemps contentées d'examiner la rentabilité des organisations de microfinance. Il faut attendre 2003 pour qu'une étude de l'Asociación de Instituciones Financieras para el Desarrollo Rural (FINRURAL) ${ }^{20}$ s'intéresse à l'impact de la microfinance sur ses clients: les résultats montrent des effets positifs, en particulier en termes d'augmentation des ventes et du nombre d'emplois générés. Le potentiel de la microfinance en Bolivie est considérable: les microentreprises (moins de cinq personnes) occupent les trois quarts de la maind'œuvre bolivienne et apparaissent comme un «secteur refuge» pour l'emploi. Mais cette frange de l'économie bolivienne reste fragile: diverses conditions structurelles adverses conduisent les petits entrepreneurs à se replier sur des stratégies individuelles à bas niveau de risque et à basse rentabilité. A l'heure actuelle, la microfinance ne permet pas de pallier de telles faiblesses. L'offre reflète les besoins propres des organisations, bien plus que ceux de leurs clients. Marquées par l'expérience de la crise du secteur financier de 1999 à 2002, les organisations de microfinance cherchent avant tout à réduire les risques encourus, imposant un rythme de remboursement rapide peu compatible avec des investissements productifs (une large partie des crédits sont donc utilisés à des fins de consommation) et tendant à se concentrer sur des microentreprises de vente au détail et de services à faible valeur ajoutée.

\section{L'impact sur la vulnérabilité: des effets positifs, parfois inattendus}

C'est ici que les effets les plus positifs sont observés, même s'il s'agit parfois d'effets inattendus. Dans la plupart des contextes de pauvreté, les difficultés essentielles proviennent de l'irrégularité et de l'imprévisibilité des revenus et de l'inadéquation entre revenus et dépenses. Le moindre aléa (plusieurs journées consécutives non travaillées, dépense imprévue pour raison de santé ou de décès par exemple) plonge les familles dans des situations d'endettement et de dépendance conduisant à de véritables trappes à pauvreté. Si la microfinance a souvent du mal à agir sur le montant des revenus, elle peut permettre aux familles pauvres de desserrer des contraintes de liquidité, de stabiliser et diversifier les sources de revenu, de renforcer un patrimoine productif souvent dérisoire et, ce faisant, de rendre les familles moins vulnérables. Pour diverses raisons (fongibilité des différentes sources de revenu, usage des services parfois autre que celui attendu, évolution de l'environnement externe), il est très difficile d'avoir une idée précise des impacts de la microfinance: seules des études très fines décortiquant en détail la complexité et la subtilité des stratégies financières des familles permettent des résultats fiables. Les deux exemples ci-dessous mettent en évidence cette complexité et les effets fragiles et contrastés (souvent un

20 FINRURAL, Impactos Agregados de las Microfinanzas en Bolivia, Bolivie, 2003. 
mélange d'effets positifs et négatifs) de la microfinance sur la lutte contre la vulnérabilité. Un troisième exemple présente quant à lui les espoirs d'une innovation importante dans la gestion des risques climatiques.

\section{L'élargissement des opportunités financières - effets directs et indirects du microcrédit: un exemple indien}

L'étude longitudinale, citée plus haut, auprès de travailleurs journaliers ruraux du sud de l'Inde met en évidence des résultats ambivalents en matière de lutte contre la vulnérabilité. Certaines améliorations sont indéniables mais sont elles-mêmes à l'origine de nouvelles formes de risque. Une première amélioration observée est la diminution de la part des crédits auprès de prêteurs privés pour $48 \%$ des ménages (contre $20 \%$ pour lesquels cette part s'est accrue). Du fait de taux d'intérêt moins onéreux et d'une pression plus forte au remboursement (échéancier précis obligeant les ménages à rechercher plus activement d'autres ressources), cette substitution partielle permet aux familles de réaliser des économies substantielles (mais sous la contrainte). On observe également une évolution positive du ratio endettement/patrimoine (pour $85 \%$ à $90 \%$ des familles): au fil du temps, les ménages acquièrent plus de patrimoine et/ou diminuent leur endettement (ou bien augmentent leur patrimoine plus fortement que leur endettement), ce qui les rend moins vulnérables. Outre les téléviseurs, les bicyclettes et le bétail, l'acquisition d'or représente une part importante des augmentations de patrimoine (25\% des familles ont acheté de l'or, très souvent avec les crédits de microfinance). Pour les populations pauvres de l'Inde rurale du Sud, l'or reste aujourd'hui la principale forme d'épargne. Il a l'avantage de concilier des fonctions de sécurité, de liquidité (il peut être immédiatement mis en gage car les prêteurs sur gage sont présents partout), de prestige social et enfin de spéculation (le cours de l'or en roupies a doublé entre 2002 et 2006). Par ailleurs, les produits proposés par les organisations de microfinance sont mal adaptés et sont considérés comme contraignants: les montants mais surtout les délais et les dates d'obtention des microcrédits coïncident rarement avec les besoins des familles. Quand le crédit est disponible, c'est rarement au moment où le ménage en a le plus besoin. Aussi une partie ou la totalité du microcrédit est-elle utilisée pour acheter de l'or, qui sera ensuite gagé lorsqu'un besoin se présentera. Au final, la microfinance permet une augmentation de l'éventail des opportunités financières disponibles, directement, via les microcrédits, mais aussi indirectement, via l'or et les prêts sur gage, et via une meilleure solvabilité auprès de créanciers potentiels, qui savent que leur débiteur pourra solliciter un microcrédit s'il a du mal à rembourser. Cet élargissement des opportunités financières permet aux familles de mieux gérer leur trésorerie et de pallier plus facilement les différents aléas de la vie quotidienne, mais il est également source de risque: entre $30 \%$ et $45 \%$ des familles sont désormais plus endettées qu'auparavant. Entre $10 \%$ et $15 \%$ ont vu le ratio endettement/capital diminuer et sont donc plus vulnérables. Les autres ont acquis plus de capital qu'elles n'ont augmenté leur dette, mais l'augmentation du capital reste précaire car elle est en partie liée à la valeur de l'or, ou à la santé (fragile) du bétail (capital «élevage»). 


\section{L'effet du microcrédit sur la gestion \\ des contraintes de liquidité dans le temps: un exemple mexicain}

Les recherches menées dans le contexte du Mexique rural décrites précédemment aboutissent à des résultats comparables à ceux mis en évidence dans le contexte indien. Le principal effet du microcrédit se situe également au niveau de la gestion intertemporelle de la liquidité. L'apport en liquidité que constitue le microcrédit permet à de nombreuses familles de réduire le décalage temporel entre les revenus et les dépenses. Nombre d'entre elles, nous l'avons évoqué plus haut, ont émigré en partie aux Etats-Unis; or cette spécificité conditionne fortement la manière dont les microcrédits sont utilisés. Pour les ménages qui reçoivent régulièrement une aide financière des membres émigrés, l'accès au microcrédit ne réduit que partiellement et pour une courte durée dans le temps la dépendance à l'égard des transferts migratoires: il constitue une anticipation sur des revenus ne résultant pas d'une activité économique locale et constituant, pour $50 \%$ des familles comptant un ou plusieurs membres émigrés, plus de $45 \%$ du revenu monétaire. Pour ces ménages, le microcrédit est essentiellement destiné aux dépenses non productives: dépenses courantes, achat de biens durables, financement de l'activité agricole destinée à l'autosubsistance de la famille. On constate par ailleurs que pour ces familles la stratégie privilégiée pour le remboursement du microcrédit n'est autre que le recours aux transferts migratoires: $43 \%$ des emprunteurs remboursent tout ou partie du microcrédit avec l'argent de la migration, soit près de $100 \%$ des emprunteurs recevant des transferts.

En dehors du contexte de forte migration internationale, le remboursement du microcrédit est crucial car il conditionne la continuité du droit d'accès à la source de liquidité procurée par le dispositif de microfinance dans le futur. Or, cette continuité est assurée par le recours à l'emprunt informel ou par l'adoption d'arrangements tacites entre membres d'un même groupe solidaire: il arrive fréquemment que l'offre de microfinance ne coïncide pas pleinement avec les besoins (montants, moment de l'octroi) des emprunteurs. Ces derniers adoptent alors des stratégies leur permettant de contourner les rigidités de l'offre. Ainsi, dans plus de $12 \%$ des groupes observés, l'une des stratégies consiste à ce que l'emprunteur dont les besoins sont inférieurs au montant proposé par le dispositif de microfinance (au vu des performances de remboursement des prêts antérieurs) emprunte un montant plus élevé que ses besoins et reprête la différence ou la totalité du microcrédit à un autre membre du groupe. Cette stratégie traduit le souci de ne pas «gâcher» cette liquidité ni pour soi-même ni pour les autres en cas de décalage entre l'offre et ses besoins propres.

\section{Un exemple d'innovation: I'assurance sécheresse en Inde}

Lutter contre la vulnérabilité, c'est aussi faire preuve d'innovation. Ici, les résultats du secteur de la microfinance sont encourageants dans la mesure où des limites jusqu'à présent infranchissables sont repoussées. C'est le cas par exemple avec la microassurance sécheresse, une innovation majeure en termes de gestion des risques climatiques, qui est née récemment en Inde ${ }^{21}$. Bénéficiant

${ }^{21}$ Voir: C. Fouillet, Construction sociale, économique et spatiale de la microfinance: l'exemple de l'Inde, thèse de doctorat en sciences économiques et sociales, Université Lumière Lyon 2, à paraître. 
de l'existence de produits financiers dérivés ${ }^{22}$ sur les marchés internationaux, d'incitations des autorités bancaires et du savoir-faire d'institutions de microfinance intervenant en milieu rural, cette innovation permet de repousser les limites de l'assurabilité des petites exploitations agricoles indiennes situées en zones sèches. Les risques en agriculture et notamment les risques liés à un déficit de pluie (une faible mousson par exemple) ne sont pas nouveaux : les agriculteurs, les banques rurales et les prêteurs au sens large ont toujours développé des stratégies pour réduire ces risques et en gérer les conséquences, principalement une baisse des revenus. Mais ces stratégies se sont toujours butées au caractère covariant des risques climatiques, c'est-à-dire affectant l'ensemble de la population d'une zone donnée.

Les produits d'assurance basés sur un indice climatique proposent une alternative à ce problème de risque covariant. L'innovation principale réside dans le fait que l'assurance et les remboursements liés à des aléas naturels sont définis en fonction d'une variable exogène comme la mesure des précipitations, des températures ou encore de la vitesse du vent. Ces mesures, enregistrées à un niveau régional ou local par des stations météorologiques, sont indépendantes des actions des fermiers et observables par les deux parties, permettant ainsi de s'affranchir des problèmes d'asymétries informationnelles. L'assureur fait alors correspondre ces indices avec un certain niveau de pertes pour une culture donnée s'il y a lieu. Dans le cas de la microassurance sécheresse, les indemnités sont calculées en fonction du niveau de précipitation, de la surface cultivée et du type de culture. Les remboursements sont ainsi étroitement corrélés avec les variations du niveau de revenu de l'exploitation agricole assurée et ne dépendent pas d'une appréciation au cas par cas.

Ensuite, les services financiers basés sur un indice climatique facilitent le transfert des risques sur les marchés financiers où les investisseurs peuvent acquérir ces contrats comme n'importe quel investissement dans un portefeuille diversifié. Les produits financiers dérivés constituent de nouveaux instruments de transfert des risques climatiques. Une sécheresse en Inde n'est pas un risque assurable au niveau du marché indien car il revêt un caractère non diversifiable qui, à une autre échelle, asiatique ou mondiale par exemple, peut disparaître et le rendre assurable. De plus, en couvrant principalement l'investissement productif, cette microassurance permet d'éviter les reports d'endettement d'une saison à l'autre.

Même s'il est encore trop tôt pour évaluer son impact, on peut supposer que la microassurance sécheresse aura un effet moteur permettant de limiter les pertes de revenus dues aux aléas climatiques et d'inciter les agriculteurs à investir davantage dans des productions à plus forte valeur ajoutée (par exemple, l'arachide ou le coton à la place du mil ou du sorgho).

22 Les produits dérivés sont des produits financiers dont la valeur dépend de l'évolution d'un indice nommé sous-jacent (précipitations, températures, vitesse du vent, etc.). 
La microfinance peut être un filet de sûreté pour les plus démunis, mais elle ne peut qu'exceptionnellement être un facteur de lutte contre la pauvreté par accroissement des revenus et diminution des inégalités. En l'état présent de l'offre, elle aurait plutôt tendance à aggraver certaines formes d'inégalités. Les débats actuels, malheureusement, considèrent pour acquise la contribution positive de la microfinance à l'éradication de la pauvreté. Le thème du refinancement bancaire et commercial des organisations, par ailleurs tout à fait légitime et pertinent quand il est à sa place, en vient à occuper l'essentiel des débats, occultant une question plus déterminante pour l'avenir du secteur: celle de la nature des services. Ils sont encore très largement inadaptés à une demande extrêmement complexe et diversifiée, et ceci vaut tant pour la microfinance commerciale que pour celle ayant des objectifs immédiatement sociaux. Les articles, les conférences et les ouvrages se multiplient sur la question du refinancement des institutions de microfinance et de leur accession aux marchés privés afin d'élargir leur potentiel - cette fameuse idée du building inclusive financial system. Les plus importantes d'entres elles embauchent des spécialistes en marketing pour développer des campagnes de promotion de prêts et d'assurances en direction de ces pauvres supposés potentiellement rentables. Mais s'inquiète-t-on de façon rigoureuse et systématique de savoir si les clients sont en mesure d'absorber ces nouveaux prêts et de les rembourser? On a toute raison d'en douter. A l'heure actuelle, trois enjeux nous semblent essentiels pour l'avenir de la microfinance. Le premier consiste à remettre le client et la qualité des services au cœur des débats. Paradoxalement, le bien-être des pauvres est finalement oublié au profit de la survie des organisations ellesmêmes. Améliorer la qualité des services, nous l'avons déjà mentionné, suppose d'innover. L'innovation existe, mais elle reste limitée à une minorité d'organisations. Enfin, la régulation du secteur est également une priorité. Réguler, cela signifie mettre en place des instances de concertation et de coordination entre les différents prestataires de services et les différents bailleurs de fonds, adopter une déontologie pour éviter les dérives «usurières ${ }^{23}$, fixer des règles claires mais aussi compatibles avec une offre de qualité (et pas uniquement avec des contraintes de rentabilité financière des organisations). Ici encore, des initiatives de régulation existent, mais elles se révèlent très largement insuffisantes. Dans de nombreux pays (Etats en Afrique de l'Ouest et en Afrique centrale, Inde, etc.), ce sont les banques centrales qui ont pris l'initiative de la régulation, souvent en réaction à un certain nombre de dérives ou de banqueroute d'IMF. Elles s'intéressent essentiellement aux aspects purement bancaires de la régulation (protection de l'épargne, sécurisation des fonds, transparence de la comptabilité). Les aspects déontologiques, la protection contre les concurrences déloyales ou la chasse aux «moutons noirs», se mettent également en place, mais sur l'initiative d'associations d'IMF qui souvent regroupent essentiellement les plus importantes ou les plus actives. Ces dernières se

23 Voir: M. Roesch, I. Guérin and C. Fouillet, Debt, Debt Burden and Interest Rates: Has «the Microfinance Machine» Gone Mad? Crisis in Andhra Pradesh (India), Working Paper, French Institute of Pondicherry, 2006. 
muent en groupes de pression auprès des parlementaires quand il s'agit de légiférer sur le secteur (mise en place des «statuts » IMF, régulation des taux usuraires, etc.). L'ensemble de ces initiatives est souvent embryonnaire et peu professionnel, car rares sont les pays disposant d'un arsenal complet et cohérent de règles applicables à la microfinance. La «jeunesse» du secteur est flagrante dans ce domaine.

\section{Bibliographie}

Arredondo Casillas, E., Estudio para el Desarrollo y Fortalecimiento de Instituciones Financieras Rurales en las Regiones Marginadas de Oaxaca, SAGARPA; Banco mundial, 2001.

Groupe consultatif d'assistance aux pauvres (CGAP), «La coordination entre bailleurs de fonds à l'intérieur d'un pays», in I. Guérin et J.-M. Servet (dir.), Exclusion et liens financiers. Rapport du Centre Walras 2003, Paris, Economica, 2003, pp. 342-361.

Daley-Harris, S., Etat de la campagne du sommet du microcredit. Rapport 2006, 2006, <http://www.

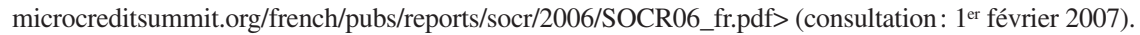

Doligez, F., «Microfinance et dynamiques économiques: quels effets après dix ans d'innovations financières?», Revue Tiers Monde, vol. 43, n 172, 2002, pp. 783-809.

Fernando, J.L. (ed.), Microfinance: Perils and Prospects, London, Routledge, 2006.

Fouillet, C., Construction sociale, économique et spatiale de la microfinance: l'exemple de l'Inde, thèse de doctorat en sciences économiques et sociales, Université Lumière Lyon 2, à paraître.

—, La microfinance serait-elle devenue folle? Crise en Andhra Pradesh (Inde), Bulletins d'information du mardi (BIM), Pôle microfinancement (CIRAD/GRET), 25 avril 2006.

Fouillet, C., I. Guérin, S. Morvant-Roux, M. Roesch et J.-M. Servet, «Le microcrédit au péril du néolibéralisme et de marchands d'illusions. Manifeste pour une inclusion financière socialement responsable», Revue du MAUSS, à paraître.

Gentil, D. et J.-M. Servet (dir.), «Microfinance: petites sommes, grands effets?», Revue Tiers Monde, vol. 43, nº 172, 2002, pp. 729-890.

Guérin, I., «Women and Money: Multiple, Complex and Evolving Practices », Development and Change, vol. 37, nº 3, 2006, pp. 549-570.

Guérin, I., C. Fouillet et J. Palier, «La microfinance indienne peut-elle être solidaire?», Revue Tiers Monde, à paraître.

Guérin, I., K. Marius-Gnanou, T. Pairault et J.-M. Servet (dir.), La microfinance en Asie. Entre traditions et innovations, Khartala, Paris, 2005.

Guérin, I. et J. Palier (dir.), Microfinance Challenges: Empowerment or Disempowerment of the Poor?, coll. Sciences sociales, $n^{\circ} 10$, Pondichéry, Institut français de Pondichéry, 2005.

Hulme, D. and P. Mosley, Finance against Poverty, vol. 1, London, Routledge, 1996.

Lapenu, C. and M. Zeller, Distribution, Growth, and Performance of Microfinance Institutions in Africa, Asia, and Latin America, FCND Discussion Paper, $\mathrm{n}^{\circ} 114$, Washington, International Food Policy Research Institute, June 2001.

Martínez, O., Microfinance et territoires dans le Sud-Est béninois. Approche en termes de risque au travers d'une vision discriminante de l'espace, LPED, Marseille, Université de Provence Aix-Marseille 2, à paraître, $13 \mathrm{p}$.

Morduch, J., «The Microfinance Promise», Journal of Economic Literature, vol. 37, n 4, 1999, pp. 15691614.

Morvant-Roux, S., Processus d'appropriation des dispositifs de microfinance: un exemple en milieu rural mexicain, thèse de doctorat en sciences économiques, Université Lumière Lyon 2, 2006.

Roesch, M., Des dettes jusqu'à ne plus en vivre, Bulletins d'information du mardi (BIM), Pôle microfinancement (CIRAD/GRET), 11 avril 2006.

Roesch, M., I. Guérin and C. Fouillet, Debt, Debt Burden and Interest Rates: Has «the Microfinance Machine» Gone Mad? Crisis in Andhra Pradesh (India), Working Paper, French Institute of Pondicherry, 2006.

Roesch, M., I. Guérin, S. Kumar, M. Thanuja, Venkatasubramanian and Ponnarasu, Impact Assessment of the Peblisa Project in Tamil Nadu, Report for the International Labour Organisation, Pondicherry, French Institute of Pondicherry, 2006. 
Schreiner, M. and R. Vonderlack, «Women, Microfinance and Savings : Lessons and Proposals », Development in Practice, vol. 12, $\mathrm{n}^{\circ}$ 5, 2002, pp. 602-612.

Servet, J.-M., Banquiers aux pieds nus. La microfinance, Paris, Odile Jacob, 2006.

- (dir.), Epargne et liens sociaux. Etudes comparées d'informalités financières, Paris, Association d'économie financière, 1995.

Simanovitz, A. and A. Walter, «Ensuring Impact: Reaching the Poorest while Building Financially Selfsufficient Institutions, and Showing Improvement in the Lives of the Poorest Women and Their Families », in S. Daley-Harris (ed.), Pathways out of Poverty: Innovations in Microfinance for the Poorest Families, Bloomfield, CT, Kumarian Press, 2002, pp. 1-90.

Stiles, K., «International Support for NGOs in Bangladesh: Some Unintended Consequences », World Development, vol. 30, n 5, 2002, pp. 835-846.

Vogelgesang, U., «Microfinance in Times of Crisis: The Effects of Competition, Rising Indebtedness, and Economic Crisis on Repayment Behavior», World Development, vol. 31, n 12, 2003, pp. 2085 2114.

Wampfler, B., Les conventions de microfinance: un outil de développement socialement durable. Une approche institutionnaliste, mémoire de travaux en vue de l'habilitation à diriger les recherches, Université de Saint-Quentin-en-Yvelines, 2004. 